\title{
Unraveling the Mechanism Underlying Surface Ligand Passivation of Colloidal Semiconductor Nanocrystals: A Route for Preparing Advanced Hybrid Nanomaterials
}

Meghan B. Teunis, ${ }^{1}$ Thakshila Liyanage, ${ }^{1}$ Sukanta Dolai, ${ }^{1}$ Barry B. Muhoberac, ${ }^{1}$ Rajesh $\operatorname{Sardar}^{*, 1,3}$, and Mangilal Agarwal ${ }^{*, 1,2,3}$

${ }^{1}$ Department of Chemistry and Chemical Biology, Indiana University-Purdue University Indianapolis, 402 N. Blackford Street, Indianapolis, Indiana 46202, United States

${ }^{2}$ Department of Mechanical Engineering, Indiana University-Purdue University Indianapolis, 723 W. Michigan Street, Indianapolis, Indiana 46202, United States

${ }^{3}$ Integrated Nanosystems Development Institute, Indiana University-Purdue University Indianapolis, 755 W. Michigan Street, Indianapolis, Indiana 46202, United States

This is the author's manuscript of the article published in final edited form as:

Teunis, M. B., Liyanage, T., Dolai, S., Muhoberac, B. B., Sardar, R., \& Agarwal, M. (2017). Unraveling the Mechanism Underlying Surface Ligand Passivation of Colloidal Semiconductor Nanocrystals: A Route for Preparing Advanced Hybrid Nanomaterials. Chemistry of Materials, 29(20), 8838-8849. https://doi.org/10.1021/acs.chemmater.7b03240 
Abstract: Optically bright colloidal semiconductor nanocrystals (CSNCs) are important nanomaterials because of their potential applications such as cellular imaging and solid-state lighting. The optoelectronic properties of CSNCs are strongly controlled by the chemical nature of the surface passivating ligands that are introduced during their synthesis. However, the existing LaMer growth model does not provide a clear understanding of the stage when ligands become attached onto the CSNCs surface. Herein, apart from the three stage formation mechanism of CSNCs (supersaturation, nucleation, and growth), an entirely new stage - solely involving surface ligand attachment onto fully grown CSNCs - is now reported that controls their photoluminescence properties. Furthermore, we also demonstrate a fundamentally new surface modification approach using partially passivated CSNCs to introduce a variety of functional groups (azide, alkene, and siloxane), including photoisomerizable molecular machines (e.g., azobenzene), without the use of “state-of-the art” ligand exchange chemistry. Knowledge of the ligand adsorption phenomena and resulting adsorption time-dependence expands our fundamental understanding of structure-property relationships while allowing us to engineer novel hybrid functional nanomaterials with both previously unknown optoelectronic properties and supermolecular assembly options for various applications. 


\section{INTRODUCTION}

Colloidal semiconductor nanocrystals (CSNCs) display size-, shape-, and surface ligand chemistry-dependent optoelectronic properties, ${ }^{1-8}$ and because of their unique properties that are strongly dependent on such structural parameters, CSNCs have been widely studied for both fundamental reasons and device applications. ${ }^{9-18}$ Considering the large number of procedures available in the literature for CSNCs synthesis, it is surprising that their formation mechanism is still poorly understood. The solution formation of CSNCs coated with surface passivating ligands is considered a three stage process (supersaturation, nucleation, and growth), which follows the LaMer Model. ${ }^{19}$ However, the model fails to provide a quantitative understanding of ligand adsorption onto the CSNC surface. In this context, the following questions in colloidal CSNCs synthesis have not been addressed before and thus create a large void in our scientific understanding: (i) At what stage during the formation of CSNCs is their surface fully passivated by ligands? (ii) What is the complete model of in-situ ligand adsorption? (iii) How does ligand chain length control the adsorption kinetics? (iv) It is feasible to introduce chemical functionalities on the surface of CSNCs without the traditional ligand exchange procedure?

Surface ligands are an integral part of CSNC synthesis through controlling their growth process and size distribution, as well as in maintaining solution dispersibility and colloidal stability. These ligands also have a central role in controlling CSNC

photoluminescence (PL) and charge transfer properties. Thus, answering these questions will not only advance our basic scientific knowledge of surface ligand chemistry-dependent optoelectronic properties, but also enhance the ability to prepare novel hybrid functional nanomaterials for advanced technological applications. 
The PL emission peak shape and quantum yield of CSNCs are controlled by the surface ligand chemistry, ${ }^{20-22}$ where incomplete surface passivation results in formation of nonradiative trap states which induces a broad PL emission peak and low quantum yield. Additionally, from the time-dependence of PL during CSNC formation, it had been postulated $^{23,24}$ that the narrowing of the PL peak is due to "size focusing" leading to formation of nearly monodispersed, ligand-passivated CSNCs during the growth stage of the LaMer model (Scheme 1). ${ }^{19,}{ }^{25}$ However, the narrowing of the PL peak may also be caused by alteration of the CSNC core composition or by passivation of the majority of the surface sites without size focusing, which is an alternative mechanism that has not been considered before. The traditional CSNC formation model relies on simultaneously adsorption/desorption of surface ligands throughout the nucleation and growth processes. However, the model does not provide information about the in-situ ligand adsorption onto fully-grown CSNCs in solution where nucleation and growth processes have been completely terminated. Moreover, in current high temperature synthetic methods, the entire formation process (including nucleation and growth) takes place over the time scale of seconds to a few minutes and optical measurements are unable to determine the percentage of the surface that is passivated by ligands during the growth stage. Therefore, intriguing information involving the role of surface passivating ligands in the nucleation and growth stages of CSNCs formation is currently unavailable but will potentially enhance the quality of CSNCs synthesis and their optoelectronic properties.

Herein for the first time we show that in addition to the three stage LaMer model for CSNC formation, an additional stage (stage IV, surface ligation) is present involving solely CSNC surface passivation by ligands (see Scheme 1). This unique demonstration is achieved by combining matrix-assisted laser desorption ionization time-of-flight mass spectrometry (MALDI-TOF-MS) with time-dependent photophysical analysis and quantitative determination of surface ligand population by various analytical methods during and after the 
nucleation and growth stages. We show the strong interplay between the ligand chain length and speed of adsorption, which results in a narrowing of the PL emission peak without compromising the CSNC core composition. We also establish a model for adsorption of ligands onto colloidal CSNCs, which is found to follow a Langmuir isotherm. Finally, a fundamentally unique surface functionalization technique is demonstrated for the first time to introduce a variety of specific functionalities [e.g., azide, alkene, azobenzene (AB), and siloxane] without the use of "state-of-the art" ligand exchange chemistry.

\section{RESULTS AND DISCUSSION}

Time-Dependent Spectroscopy and Spectrometry Characterization of CSNCs Formation.

To elucidate the general mechanism of surface ligand passivation we studied magic-sized (CdSe) ${ }_{33 / 34}$ CSNC formation as a model system because the CSNCs have : (i) atomically precise core composition, (ii) unusually high stability in solution, ${ }^{26-29}$ and (iii) well-defined absorption and PL emission spectral characteristics. ${ }^{30,31}$ The room temperature (CdSe) $)_{33 / 34}$ CSNC synthesis was conducted by modification of our previously published procedure to reduce the rate of synthesis. ${ }^{32}$ In this study we used oleylamine (OLA) as the surface passivating ligand to expand the complete formation process to $\sim 24 \mathrm{~h}$ so that the mechanism underlying the ligand adsorption process could be studied through time-dependent spectroscopy and mass spectrometry techniques.

Figure 1A and Supporting Information Figure S1 illustrate UV-vis absorption spectra of the formation of CdSe CSNCs acquired from the crude reaction mixture diluted with toluene. A continuous red-shift of the absorption peak along with peak narrowing was observed (Figure 1A inset, $\bullet$ ) until 90 min and at this time a stable peak at $418 \mathrm{~nm}$ was detected. Over the course of another $\sim 150 \mathrm{~min}$, the peak intensity increased (Figure 1A inset,

ש) without any further shift in the peak position. The extremely narrow peak at $418 \mathrm{~nm}$ with 
full-width at half maxima of $12 \mathrm{~nm}$ suggests that the reaction mixture contained almost exclusively single-sized (CdSe) ${ }_{33 / 34}$ CSNCs. ${ }^{32-34}$ Based on this absorption peak characteristic, we propose that under our experimental conditions the nucleation and growth processes of (CdSe) ${ }_{33 / 34}$ CSNCs formation were completed by 240 min with stage III occurring by the continuous growth model. ${ }^{35,36}$

To further characterize the nucleation and growth processes, time-dependent MALDITOF-MS analysis (Figure 1B and Figure S2), which was first introduced by Whetten and cororkers ${ }^{37}$ for the characterization of Au nanoparticles, was conducted in which purified sample was mixed with the commonly-used matrix trans-2-[3-(4-tert-Butyl-phenyl)-2-methyl2-propenylidene]malononitrile (DCTB). Although MS was used to investigate $\mathrm{Au},{ }^{38-40} \mathrm{InP},{ }^{41}$ and $\mathrm{Fe}_{2} \mathrm{O}_{3}{ }^{42}$ nanoparticle formation, to the best of our knowledge, ours is the first example where CdSe CSNC formation was studied using the MS technique. Until 90 min of growth, our MALDI-TOF-MS data of the isolated and purified samples showed a broad mass distribution of CdSe CSNCs that became narrower over the next $150 \mathrm{~min}$. Finally at $240 \mathrm{~min}$, two sharp peaks at m/z 6506.5 and 6314.7 appeared that correspond to the presence of (CdSe) 34 and (CdSe) $)_{33}$ CSNCs, respectively, and they retained their shape continuously for an additional $18 \mathrm{~h}$ of synthesis. The MS data are supported by UV-vis analysis in which the lowest energy absorption peak position and intensity remained constant (see Figure 1A, inset). Previously, through use of variable laser intensity in MALDI-TOF-MS analysis, we demonstrated exclusive formation of these two compositions of CdSe CSNCs under our experimental conditions. ${ }^{32}$ It is important to mention that due to weak interaction between surface Cd and amines, all of the OLA ligands were detached from the surface of the CdSe CSNCs during the MALDI-TOF-MS analysis, as previously demonstrated by Buhro and coworkers. ${ }^{43}$ Nevertheless, our time-dependent MS analysis suggests that at the beginning of the reaction, ultrasmall CdSe CSNCs of various atomic compositions were formed that transformed into nearly single-sized magic composition (CdSe) $33 / 34$ SNCs by the continuous 
growth model, which occurs during stage III. ${ }^{19}$ Furthermore, the MALDI-TOF-MS analysis nicely corroborates the UV-vis absorption data in terms of nucleation and growth time, which altogether takes place within 240 min of the reaction initiation.

We characterized our observed changes in the photoluminescence (PL) properties of the CdSe CSNCs acquired from the crude reaction mixture diluted with toluene into different time intervals (Figure 1C and Figure S3). Initially a trap-state mediated, broadband PL peak appeared at $\sim 500 \mathrm{~nm}$. Over time the intensity of the peak decreased followed by appearance of a band-edge peak along with the broadband peak. At the end of the synthesis (1440 min),

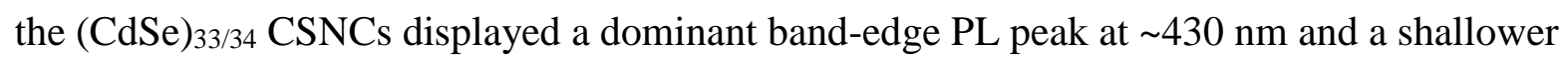
broadband emission covering 450-600 nm. The final PL characteristic corroborates our previous work and suggests the formation of (CdSe) ${ }_{33 / 34}$ CSNCs. ${ }^{32}$ Because OLA is a L-type ligand and can only passivate surface Cd sites, surface Se sites remain unpassivated resulting in formation of surface trap states and the appearance of shallow broadband emission in the PL.

The changes in the PL spectral peak positions and overall shape during the formation of CdSe CSNCs can be explained based on the CdSe core composition and surface ligand chemistry characterized by our UV-vis spectroscopy and the MALDI-TOF-MS analyses. Firstly, during the initial 30 min the red-shift (from 503 to $515 \mathrm{~nm}$ ) of the broadband PL peak (Figure 1D, $\mathbf{\square}$ ) was governed by an increase in number of CdSe units forming the inorganic core. This result is also in agreement with the UV-vis analysis in which a nearly $10 \mathrm{~nm}$ redshift in the lowest energy absorption peak was observed. Furthermore, the broad PL peak is a characteristic of surface-related trap states. ${ }^{20,21}$ Secondly, a surprising observation was the blue-shift of the PL peak from 515 to $490 \mathrm{~nm}$ (Figure 1D, $\mathbf{0}$ ) during the 30 to $120 \mathrm{~min}$ interval of the reaction with the peak remaining broad. In theory, a blue shift of a PL peak should be interpreted as either (i) dissociation of larger CSNCs to smaller ones or (ii) change in the internal and/or surface trap-states of CSNCs. Our time-dependent UV-vis spectra 
(Figure 1A inset, •) exhibited no change in the lowest energy absorption peak position while the peak intensity continued to increase at $418 \mathrm{~nm}$. Taken together this suggests that dissociation of the (CdSe) $33 / 34$ core did not take place but rather CSNCs with this core composition continued to form in the reaction mixture through size focusing via continuous supplementation of monomers. Time-dependent X-ray diffraction (XRD) analysis (Figure S4) shows that peaks related to the characteristic wurtize crystal structure of the CdSe core became more defined during this period. Therefore, amorphous CSNCs were converted to a more crystalline nature. Under such circumstances, slow removal of internal as well as surface trap states that would cause a blue-shift of the PL peak is expected to occur. ${ }^{44}$ Thus, the observed blue-shift could be due to a combination of size focusing and change in the various trap states. Finally and most importantly, we observed two distinct changes in the CdSe CSNC emission properties (Figure 1C \& D) during 240 to 1440 min of the synthesis: (i) The appearance of a band-edge PL peak ( 430 nm), which became narrower over time without changing its position (Figure 1D, •), and (ii) a simultaneous increase in intensity of this bandedge PL peak while the broadband peak decreased. In an interpretation of such timedependence of PL, Peng and coworkers ${ }^{24}$ and Bullen et al. ${ }^{23}$ postulated "size focusing” of the QD core and formation of nearly monodispersed, ligand-passivated QDs during stage III of the LaMer model. However, we should point out that the size focusing and decrease in polydispersity of QDs during the growth stage in the traditional Lamer model is not fully applicable to our system, and specifically to the continuous increase in absorption intensity over a period of 150 min without any change in the absorption peak position (Figure 1A inset). This suggest that a considerable amount of monomer was still present in the colloidal solution and only a small amount of monomer was converted to CdSe CSNC during the nucleation stage when size focusing occurs via a continuous supply of monomers. Thus we previously described this stage as "continuous growth,"29 which requires a constant supply of monomers by diffusion followed by their reaction at the surface of the CdSe CSNC until a magic-sized 
composition is achieved. Therefore, the Ostwald ripening-driven mass transport/diffusion process controls the reaction at the surface, which is within the Lamer model for monodispersed CSNC formation. Nevertheless, our present work is mostly focus on characterizing a stage beyond the growth stage where only surface ligation takes place.

Perhaps it is important to note that under our reaction conditions the nucleation and growth processes were fast and decoupled because the number of nuclei was high. This in turn originates because the nucleation process was more feasible in the presence of the L-type ligand OLA, which has a weak ability to complex with Cd. Thus, in the presence of a strongly coordinating ligand such as oleic acid, the nucleation and growth rates are expected to be different. A similar argument is also valid for the reaction temperature. At lower temperature, a smaller number of nuclei are expected to form, and thus the growth rate will be slower as well. Furthermore, the amine adsorption rate is also expected to be slower. Therefore, the growth and ligand adsorption stages are expected to overlap more, and the distinct stages that we observed under our experimental conditions might not be feasible to detect. Taken together, the identification of the ligand adsorption stage after the nucleation and growth stages in CSNC synthesis requires appropriate choice of surface passivating ligand and reaction temperature.

Time-Dependent Characterization of Surface Ligand Chemistry. Our time-dependent UV-vis absorption (Figure 1A) and MALDI-TOF-MS (Figure 1B) analyses however confirm that both composition of the (CdSe) $)_{33 / 34} \mathrm{CSNC}$ core and its wurtize crystal structure remained constant during the 240 to 1440 min interval of the synthesis. The implication is that our observed changes in the PL spectra (Figure 1C) should be related to changes in the CSNC surface structure, most likely by influence of the surface passivating ligands. Therefore based on structural and optical characterization, we hypothesize that in addition to the well-establish three stages of the LaMer model for magic-sized CdSe CSNC formation, a fourth stage is 
involved consisting of slow attachment of surface passivating ligands onto fully grown CSNCs without compromising their core composition and crystallographic structure. Thus, narrowing of the PL peak apparently results from continuous attachment of OLA during the synthesis and passivation of non-radiative surface trap-states. To validate our hypothesis, we performed time-dependent X-ray photoelectron (XPS) and ${ }^{1} \mathrm{H}$ NMR spectroscopy characterizations to quantify the average number of OLAs bound per (CdSe) ${ }_{33 / 34} \mathrm{CSNC}$ during the 240-1440 min interval of the synthesis on isolated and purified samples from three independent measurements. Both $\mathrm{XPS}^{45,46}$ and $\mathrm{NMR}^{47,48}$ techniques are commonly used for surface ligand quantification of CSNCs. Figure 2A and Figure S5 show XPS spectra of the Cd 3d and N 1s regions. Observing the two sharp Cd 3d peaks suggests no formation of surface $\mathrm{CdO}^{9,45}$ during the extended time period of synthesis. Moreover, the $\mathrm{Cd}$ :Se ratio was found to be 1:1 also in agreement with the stoichiometric (CdSe) $33 / 34$ core. The energy dispersive spectroscopy (EDS) analysis confirmed the Cd:Se ratio of 1:1 (data not shown). Because the core composition did not change over the above-mentioned time period, we determined the concentration of $\mathrm{N}$ over time based on the ratio of $\mathrm{N}$ :Cd. Under our experimental conditions, OLA is the only source of nitrogen used in the synthesis, and thus we can calculate the number of OLAs per (CdSe) $33 / 34$ core, as presented in Figure 2D (black diamonds). The same samples were also analyzed by ${ }^{1} \mathrm{H}$ NMR (Figure 2B) using ferrocene as an internal standard to quantify the number of OLAs attached per (CdSe) ${ }_{33 / 34} \mathrm{CSNC}$. The broad vinyl proton ($\mathrm{CH}=\mathrm{CH}-$ ) signal at $\sim 5.3 \mathrm{ppm}$ suggests that all the OLAs were attached onto the surface of (CdSe) ${ }_{33 / 34}$ CSNCs (Figure 2C). ${ }^{49}$ Most importantly, the NMR data are in very good agreement with our XPS results. A maximum of 19 OLAs/(CdSe) ${ }_{33 / 34}$ CSNC were attached after 1440 min (Figure 2D), which is a number well in agreement with the report by Buhro and coworkers where elemental analysis was used for ligand quantification of (CdSe) 33/34 $_{3 / 3}$ CSNC. ${ }^{33}$ Importantly as expected, at 240 min $~ 6$ OLA were attached to the surface and that number steadily increased over time. OLA is a L-type ligand and only binds to surface Cd 
sites, which are a maximum of 28 for $\left.(\mathrm{CdSe}){ }_{34}\right) .{ }^{34}$ Thus at $24 \mathrm{~h}$ of reaction, $\sim 8 \mathrm{Cd}$ along with all 28 surface Se sites remained unpassivated, which together resulted in a shallow broadband emission covering 450-600 nm (Figure 1D).

Kinetic Analysis of Ligand Adsorption. The experimental data (Figure 2D) presented above demonstrate that at 240 min of growth the core was fully formed, but only $22 \%$ of the (CdSe) ${ }_{33 / 34}$ CSNC surface was passivated with OLA. Therefore, one should expect that isolation of these partially passivated (CdSe) ${ }_{33 / 34} \mathrm{CSNCs}$ followed by treatment with the same ligand type would result in a transition from broadband PL to a nearly band-edge PL peak, which has not been demonstrated before but is now shown in Figure 1C. Moreover, we expect that shorter chain length ligands will passivate the surface more quickly because of faster diffusion through the existing ligand monolayer on the CSNC surface causing a change from broadband to band-edge emission. Furthermore, the extent of the PL transition should depend on the number of surface-bound ligands (concentration of ligand) per CSNC, and thus the ligand adsorption constant $\left(K_{a}\right)$ can be determined by fitting the data using a Langmuir isotherm (Eq. 1): $:^{50,51}$

$$
\theta=\theta_{\max } \frac{K_{a}[\text { Amine }]}{1+K_{a}[\text { Amine }]}
$$

Here $\theta_{\max }$ is the maximum surface coverage, which corresponds to the highest band-edge PL peak intensity, and $\theta=\left(I_{\theta}-I_{0}\right) /\left(I_{F}-I_{0}\right)$ is the fraction of coverage at a particular solution amine concentration, [Amine]. ${ }^{51} I_{\theta}$ is the measured PL intensity at a particular amine concentration, and $I_{F}$ and $I_{0}$ are the PL intensities at maximum and minimum surface coverage, respectively (see Figure S6A and S7). Our model is based on the assumption that (CdSe) $33 / 34$ CSNCs core composition remained constant during the post-synthetic OLA treatment and that 
at the highest amine concentration $(5 \mathrm{mM})$ we achieved maximal surface coverage $\left(\theta_{\max }=1\right)$ after $24 \mathrm{hr}$ incubation.

Figure 3A shows UV-vis and PL spectra of purified, partially OLA-passivated (CdSe) $)_{33 / 34}$ CSNCs after 240 min of the reaction, and then following ex-situ addition of $5 \mathrm{mM}$ OLA with stirring for $48 \mathrm{~h}$ at room temperature (see Supporting Information for detail). No change in the UV-vis spectrum was observed suggesting the (CdSe) ${ }_{33 / 34} \mathrm{CSNC}$ core remained unchanged after ex-situ OLA treatment, while the broadband PL peak transformed into the band-edge PL peak, indicating reduction of trap-states through surface passivation. This result strongly supports our hypothesis of a fourth LaMer stage that involves the slow attachment of surface ligands onto fully-grown CSNCs without compromising their core composition. We plotted surface coverage as a function of OLA solution concentration and from the data fit to Eq. 1 (Figure 3B, ๑), $K_{a}$ was determined to be $2.1 \times 10^{4} \mathrm{M}^{-1}$. This value is well in agreement with a literature report ${ }^{51}$ analyzing amine adsorption onto the surface of CdSe CSNCs. We also prepared partially octylamine (OCA)- and decylamine (DCA)-passivated (CdSe) 33/34 $^{3}$ CSNCs and performed ex-situ treatment with the same two ligands of varying concentrations to determine their $K_{a}$ values, which were $2.9 \times 10^{4}$ (Figure 3B, $\mathbf{A}$ ) and $2.7 \times 10^{4} \mathrm{M}^{-1}$ (Figure 3B, $\mathbf{})$, respectively. The Supporting Information Figures S6 and S7 provide additional timeand concentration-dependent PL spectra of (CdSe) ${ }_{33 / 34}$ CSNCs for different amines. Nevertheless, with all three amines the rates of adsorption onto the surface of (CdSe) 33/34 $_{34}$ CSNC are found to be slow. We believe this is due to the weaker interaction/affinity between the hard ligand nitrogen of the amine and the soft acid $\mathrm{Cd}^{2+}$.

Though we used a simple Langmuir isotherm model to quantify the amine surface binding that directly influenced the PL properties of (CdSe) ${ }_{33 / 34} \mathrm{CSNCs}$, our experimental $K_{a}$ values also suggest that the interaction of amines with the CSNC surface depends on their chain length such that smaller ligands have higher affinity. Moreover, we observed a faster transition of broadband to band-edge PL for the shorter chain length amines (e.g., OCA) than 
the longer chain length OLA (see Figure S6B). We believe OCA passivates the surface more rapidly due to faster diffusion through the ligand monolayer already existing on the CSNC surface because of less steric repulsion. Therefore adsorption speed, which is expected to be temperature dependent, may also play a critical role in the PL properties of CSNCs in general. Furthermore, the non-linear adsorption process in our system as observed in the PL spectra also suggests an energetically favorable ligand attachment event resulting in the cooperative interaction-driven ligand adsorption onto the CSNC surface. This cooperative interaction is potentially more complementary than the ligand-ligand interactions as described in TempkinFowler model. However a more detailed analysis will require a more complex mathematical model, ${ }^{50,52}$ which is beyond the scope of this article. Still, our proposed model could perhaps be applicable to high temperature synthesis of CSNCs in general using amine as surface passivating ligands.

\section{CSNC Surface Functionalization without “State-of-the Art” Ligand Exchange}

Chemistry. Surface passivating ligands are ubiquitous in CSNCs synthesis and used to not only control the CSNC aggregation during synthesis but also to tailor their photophysical properties. Over the last three decades primary amines were found be to good passivating ligands for enhancing PL properties of CSNCs. ${ }^{3,53-55}$ However, as shown by Weiss and coworkers, aniline reduces PL yield. ${ }^{56}$ Furthermore, commonly used “native” aliphatic amines are often replaced by $\omega$-functionalize-thiolate ligands through ligand exchange chemistry to induce specific nanocrystal chemical functionalities. ${ }^{57-59}$ The ligand exchange reaction removes the original surface ligands and thus alters the photophysical properties of the CSNCs.

For the post-synthetic ligand treatment, we used OLA-passivated (CdSe)33/34 CSNCs at 240 min of the synthesis because the nucleation and growth processes of CSNC formation was completed by this time and thus the CSNC core was fully grown. However, their surface 
was only partially passivated with OLA (6 OLA/CSNC, 22\%). Thus, we expect that post synthetic ligand treatments of these partially OLA-passivated (CdSe) ${ }_{33 / 34}$ CSNCs with the same ligand type (i.e., OLA) or different amines as described below should result in formation of mixed amine-passivated CSNCs and occupation of the empty surface Cd sites. Under such circumstances a transition from broadband PL to a nearly band-edge PL peak is expected to occur because of the removal of surface trap states. As shown in Figure 4, we now demonstrate for the first time a fundamentally new surface functionalization technique that introduces various functional groups such as azide $\left(-\mathrm{N}_{3}\right)$, alkene $\left(-\mathrm{CH}=\mathrm{CH}_{2}\right)$, siloxane ($\left.\mathrm{Si}\left(\mathrm{OCH}_{2} \mathrm{CH}_{3}\right)_{3}\right)$, and $\mathrm{AB}$ at the surface of the CSNCs without the use of current state-of-the art ligand exchange chemistry. In the current method fully ligand-passivated CSNCs are treated with thiolated ligands in which an X-type sulfur binding head group replaces the original L-type amine ligands because of the strong metal-sulfur interaction as compared to metal-nitrogen. Therefore, an entirely new ligand-passivated CSNC is produced. In contrast, our post-synthetic ligand treatment method does not replace the original ligands and instead a mixed ligand-passivated CSNC forms through the attachment of the second ligand onto the empty metal sites (i.e., Cd in (CdSe) $\left.)_{33 / 34} \mathrm{CSNC}\right)$ as demonstrated in Figure 4.

We selected $-\mathrm{N}_{3}$ and $-\mathrm{CH}=\mathrm{CH}_{2}$ functional groups because of their ability to conduct "click" chemistry ${ }^{60}$ and cross metathesis, ${ }^{61}$ respectively, that will allow further CSNCs surface modification $^{62}$ with a variety of organic molecules and facilitate their potential application. The - $\mathrm{Si}\left(\mathrm{OCH}_{2} \mathrm{CH}_{3}\right)_{3}$-functionalized CSNCs can act as precursors for further attachment of various biomolecules in water, thus becoming useful for biological applications. ${ }^{63,}{ }^{64}$ Finally, $\mathrm{AB}$ is capable of undergoing photoinduce cis-trans isomerization. ${ }^{65,66}$ Importantly, one can expect delocalization of exciton (electron and/or hole) wave functions into the highly $\pi$ conjugated $\mathrm{AB}$ moiety when it is in the trans conformation but not in the cis because the $\pi$ conjugation is broken. It is known that the delocalization of exciton wave functions modulates the band-gap and optoelectronic properties of CSNCs. ${ }^{49,67}$ Our surface functionalization 
approach provides three unique characteristics: (i) Attachment of various ligands of diverse functionality through metal-amine interactions. (ii) Introduction of diverse surface functionalities during the slow surface passivation step, which makes unnecessary the ligand exchange process. (iii) Attachment of unique ligands with previously unknown optoelectronic properties. Taken together, these characteristics will allow guided assembly of unique supermolecular constructs centered on the CSNCs.

Mixed ligand passivated (CdSe) ${ }_{33 / 34}$ CSNCs were characterized by various spectroscopies and transmission electron microscopy (TEM). The purified, mixed OLA-/6azido-1-hexanamine (AHA)-passivated (CdSe) ${ }_{33 / 34} \mathrm{CSNCs}$ were analyzed by UV-visible (Figure S8A) spectroscopy and no change in the absorption peak position was observed, suggesting the composition of the (CdSe) $)_{33 / 34}$ core remained constant during our newly designed surface modification technique. Our suggestion was further supported by TEM analysis where the $1.6 \mathrm{~nm}$ diameter of partially OLA-passivated (CdSe) ${ }_{33 / 34} \mathrm{CSNCs}$ remained constant with mixed OLA-/AHA passivation (Figure S9A \&B). In the PL analysis, the broad spectrum of partially OLA-passivated CSNCs turned into a band-edge peak for mixed OLA/AHA-passivated CSNCs (Figure 4.A1), which is in agreement with our previous observation for ex-situ ligand treatment (Figure 3). The presence of $-\mathrm{N}_{3}$ groups attached to (CdSe) $33 / 34$ CSNCs was confirmed by FTIR and ${ }^{1} \mathrm{H}$ NMR spectroscopies. The mixed OLA-/AHApassivated CSNCs displayed an asymmetric $-\mathrm{N}_{3}$ stretch ${ }^{68}$ at $2080 \mathrm{~cm}^{-1}$ (Figure 4.A2) that remained constant compared to pure AHA. No change indicates that the CSNC surface was not passivated by $-\mathrm{N}_{3}$ groups, and thus only the $-\mathrm{NH}_{2}$ groups from $\mathrm{AHA}$ were bound to the surface Cd sites. ${ }^{1} \mathrm{H}$ NMR analysis also supported the attachment of AHA onto the CSNCs surface (Figure 5A). In addition, NMR analysis confirmed the presence of OLA on the CSNCs surface, suggesting mixed OLA and AHA surface ligation as expected.

The mixed OLA-/amino AB (AAB)-passivated (CdSe) ${ }_{33 / 34}$ CSNCs were also analyzed by the above-mentioned spectroscopy and microscopy techniques. The FTIR spectrum 
(Figure 5B) shows the presence of azobenzene because of the appearance of a trans $-\mathrm{N}=\mathrm{N}$ stretch at $1445 \mathrm{~cm}^{-1} .{ }^{69}$ Unlike mixed OLA-/AHA-passivated CSNCs, mixed OLA-/AABpassivated (CdSe) ${ }_{33 / 34}$ CSNCs displayed a broad PL peak (Figure 4. B1). We propose that this broadness originates from the formation of mid-gap trap states, as demonstrated by the simple MO diagram in Figure S10. Importantly, UV-vis analysis showed an $80 \mathrm{meV}$ red-shift of the lowest energy excitonic peak (reduction of band-gap) of OLA-/AAB-passivated (CdSe) 33/34 CSNCs as compared to partially OLA-passivated CSNCs (Figure 4. B2). We believe that this shift is because of delocalization of the excitonic wavefunction ${ }^{49,}{ }^{67}$ from the CSNC to the highly conjugated trans azobenzene moiety, from alteration in the local dielectric environment around the CSNC, or a combination of both, but it is not due to changes of the CSNC core diameter as confirmed by TEM analysis (Figure S9C). As mentioned above, the selection of AAB for hybrid nanomaterial synthesis was to allow tuning of the band-gap through photoisomerization of azobenzene. However, we were unable to produced in-situ cis azobenzene-passivated (CdSe) ${ }_{33 / 34}$ CSNCs under UV light (350 nm wavelength) exposure because decomposition of CSNCs was observed. It is known that CSCNs undergo fast degradation under UV light irradiation in the presence of a minute amount of oxygen and/or water. Nevertheless, to prove the intended result that photoisomerization causes azobenzene structure-dependent band-gap modulation of OLA-/AAB-passivated (CdSe) ${ }_{33 / 34}$ CSNCs will require careful experimental design and is currently under investigation.

Figure 4C and D characterize OLA-/5-Hexene-1-amine (HXA)- and OLA-/(3aminopropyl)triethoxysilane (APTES)-passivated (CdSe)33/34 CSNCs. UV-vis spectra exhibited no noticeable changes in the lowest energy excitonic peak in both cases during HXA and APTES treatments of partially OLA-passivated CSNCs, suggesting no alteration of inorganic core diameter (Figure S8D and E). This result is supported by TEM analysis where (CdSe) ${ }_{33 / 34} \mathrm{CSNCs}$ retained their average diameter during the ligand treatment and purification processes (Figure S9D and E). In both cases, PL analysis (Figure 4.C1 and D1) 
showed appearance of band-edge peaks as observation for ex-situ ligand treatment and also for mixed OLA-/AHA-passivated CSNCs. ${ }^{1} \mathrm{H}$ NMR analyses of both mixed OLA-/HXA- and OLA-/APTES-passivated (CdSe) $33 / 34$ CSNCs are shown in Figure 4. C2 and D2, respectively, and it unequivocally proved the presence of mixed surface ligation. Additional analyses are provided in the Supporting Information, see Figure S11 and S12. Overall, our demonstrated surface functionalization approach should be applicable to other type (e.g., CdS, CdTe, ZnSe, PbS, etc.) CSNCs.

\section{CONCLUSION}

In conclusion, using time-dependent optical absorption, photoluminescence, and NMR spectroscopies, as well as mass spectrometry, and X-ray diffraction, we have characterized a new stage in CSNCs formation that involves solely surface ligand attachment onto fully grown CSNCs. This stage only alters the PL properties of the resulting CSNCs but has no influenced on their excitonic peak position. Our investigation has demonstrated that narrowing of the PL peak is not due to the widely accepted model of size focusing of CSNCs during the growth stage of the LaMer model but is from attachment of surface ligands that reduces the number of trap states. Furthermore, attachment of ligands onto the CSNC surface follows a Langmuir isotherm where alkyl chain length of the ligands plays a critical role in their adsorption rate. Importantly, we have demonstrated a fundamentally new surface functionalization approach to the preparation of novel CSNCs with different surface ligands that can be used to form various supermolecular structures. Taken together, we believe our investigation will aid in the preparation of hybrid inorganic-organic functional nanomaterials with unique physicochemical and optoelectronic properties for various applications without performing complicated, state-of-the-art post-synthetic ligand exchange reactions.

\section{EXPERIMENTAL SECTION}


Materials. Cadmium acetate dihydrate (Cd (OAc) $\left.)_{2} \cdot 2 \mathrm{H}_{2} \mathrm{O}\right)(98 \%)$, oleylamine (OLA) $(70 \%)$, decylamine (DCA) (95\%), octylamine (OCA) (99\%), 1-hexanethiol (HT) (95\%), 4aminoazobenzene (AAB, 98\%) (3-aminopropyl)triethoxysilane (APTES, 99\%), 5-hexen-1amine (HXA, 97\%), 1,6-dibromohexane (96\%), sodium azide (99\%), triphenylphosphine (99\%), toluene (HPLC grade), selenium (Se, 99.99\%), acetonitrile (MeCN, HPLC grade), chloroform (HPLC grade), and hexane (95\%) were purchased from Aldrich and used without further purification. Methanol (MeOH, ACS) was purchased from Fisher Scientific. Prior to use, solvents were purged with $\mathrm{N}_{2}$ for 30 min. 6-azido-1-hexanamine (AHA) was synthesized according to the literature procedure with slight modification as described below.

Preparation of 1, 6-diazidohexane. ${ }^{70}$ 1,4-dibromohexane ( $3.2 \mathrm{~g}, 0.013 \mathrm{~mol}$ ) and sodium azide (3.4 g, $0.052 \mathrm{~mol}$ ) was dissolved in $100 \mathrm{~mL}$ of DMF and reaction mixture was refluxed for 24 hours at $100{ }^{\circ} \mathrm{C}$. The reaction was quenched by adding $30 \mathrm{~mL}$ of water. The product was extracted with diethyl ether (20 mL x 4). The organic phase was washed with $5 \%$ $\mathrm{NaHCO}_{3}$ dried over $\mathrm{Na}_{2} \mathrm{SO}_{4}$, and dried under high vaccum resulting in the viscous product 1 , 6-diazidohexane. ${ }^{1} \mathrm{H}$ NMR $\left(\mathrm{CDCl}_{3}\right): \delta(\mathrm{ppm})=3.28(\mathrm{t}, 4 \mathrm{H}, J=6.8 \mathrm{~Hz}), 1.63-1.61(\mathrm{~m}, 4 \mathrm{H})$, 1.43-1.37 (m, 4H).

Preparation of 6-Azido-1-hexanamine. ${ }^{71} 5 \mathrm{~mL}$ of ethylacetate and $9 \mathrm{~mL}$ of $5 \% \mathrm{HCl}$ were added to a solution of 1 , 6-diazidohexane $(2.1 \mathrm{~g}, 0.012 \mathrm{~mol})$ in 10.5 diethyl ether at $0{ }^{\circ} \mathrm{C}$, and then $\mathrm{PPh}_{3}$ (3.85 g, 0.014mol, 1.0 equiv) was added in small portions over $1 \mathrm{~h}$. The reaction mixture was stirred at room temperature for $30 \mathrm{~h}$ and then $20 \mathrm{~mL}$ of $1.0 \mathrm{M} \mathrm{HCl}$ solution was added. The aqueous layer was collected and washed with $\mathrm{CH}_{2} \mathrm{Cl}_{2}(20 \mathrm{~mL} \times 2)$. The resulting solution was neutralized with $6.0 \mathrm{M} \mathrm{NaOH}$ by maintaining the solution $\mathrm{pH}$ of 12 . The organic compound was extracted with $\mathrm{CH}_{2} \mathrm{Cl}_{2}(20 \mathrm{~mL} \times 4)$, washed with saturated brine solution, and then dried over $\mathrm{Na}_{2} \mathrm{SO}_{4}$. The solution was further dried under high vacuum and yielded the product 6-azido-1-hexanamine. ${ }^{1} \mathrm{H}$ NMR $\left(\mathrm{CDCl}_{3}\right): \delta(\mathrm{ppm})=3.25(\mathrm{t}, 2 \mathrm{H}, \mathrm{J}=6.9 \mathrm{~Hz}), 2.68(\mathrm{t}$, $2 \mathrm{H}, \mathrm{J}=6.8 \mathrm{~Hz}), 1.63-1.55(\mathrm{~m}, 2 \mathrm{H}), 1.48-1.41(\mathrm{~m}, 2 \mathrm{H}), 1.40-1.32(\mathrm{~m}, 2 \mathrm{H})$.

Colloidal Synthesis of Ligand-Passivated (CdSe) ${ }_{33 / 34}$ CSCNs. The synthesis was performed according to our published procedure. ${ }^{32}$ The Cd-precursor was prepared as follows: In a typical Cd-precursor preparation, $5 \mathrm{~mL}$ of OLA and $0.2 \mathrm{~g}$ of $\mathrm{Cd}(\mathrm{OAc})_{2}$ were mixed in a 100 $\mathrm{mL}$ 2-neck round bottom flask and stirred under vacuum for $15 \mathrm{~min}$. Afterwords the reaction was retuned to $\mathrm{N}_{2}$ atmosphere and $5 \mathrm{~mL}$ of toluene was added. The Se-precursor was prepared in parallel as follows: $0.06 \mathrm{~g}$ of freshly ground Se was mixed with $785 \mu \mathrm{L}$ of OLA and $215 \mu \mathrm{L}$ of HT at room temperature. This mixture was first kept under vacuum for 5 min and then placed under $\mathrm{N}_{2}$ for 5 min with constant stirring. The vacuum/ $\mathrm{N}_{2}$ cycle was repeated three times and then the reaction mixture was kept under $\mathrm{N}_{2}$ for an additional $30 \mathrm{~min}$. Finally, $1.0 \mathrm{~mL}$ of the Se-precursor was swiftly injected into the Cd-precursor. Stable absorption maxima and highest PL peak intensity was observed nearly $24 \mathrm{~h}$ after the addition of Seprecursor. We consider $24 \mathrm{~h}$ as the final time point at which the (CdSe) 33/34 $_{3} \mathrm{CSNCs}$ surface was maximally passivated with OLA. Under identical mole ratios of the reagents and the same reaction conditions, OCA- and DCA-passivated (CdSe) $)_{33 / 34}$ CSNCs were synthesized. It 
should be noted that with OCA and DCA, their stable absorption maxima and highest PL peak intensity was observed nearly 4 and $10 \mathrm{~h}$ after the addition of Se-precursor, respectively.

Ex-situ Ligand Treatment of Partially Ligand-Passivated (CdSe) 33/34 $_{34}$ CSCNs. It was found for OLA-passivated (CdSe) ${ }_{33 / 34}$ CSNCs that at 240 min after the addition of Se-precursor the SNCs solution displayed a stable absorption peak and a broad PL. At this point, further surface passivation was restricted by quenching the reaction mixture. Briefly, $0.5 \mathrm{~mL}$ (CdSe) $33 / 34$ CSNCs solution was removed from and diluted in $5 \mathrm{~mL}$ of toluene, and then a 7:3 mixture of $\mathrm{MeOH}: \mathrm{MeCN}$ was added drop-wise until the solution became turbid. The solution was then centrifuged at $7000 \mathrm{rpm}$ for $7 \mathrm{~min}$. This process was repeated once more and yielded a yellow solid. A stock solution of $0.12 \mu \mathrm{M}(\mathrm{CdSe})_{33 / 34} \mathrm{CSNCs}$ was prepared in toluene. Then OLA at various concentrations between 0.25 and $5000 \mu \mathrm{M}$ was added under $\mathrm{N}_{2}$ atmosphere. The reaction mixture was stirred for $48 \mathrm{~h}$ at room temperature. During this period absorption and emission spectra were collected at various time points. A similar ex-

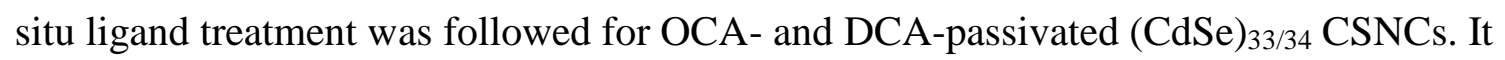

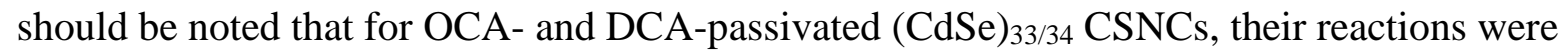
quenched at 30 and 90 min after the addition of Se-precursor, respectively. For post synthetic surface ligand modification with AHA, AAB, APTES, and HXA we followed the same procedure as used for OLA-passivated (CdSe) $33 / 34$ CSNCs.

Optical Spectroscopy Characterization. The absorption spectra were collected using a Varian-Cary 50 Scan UV-visible spectrophotometer with $1 \mathrm{~cm}$ quartz cuvettes over a range of $300-800 \mathrm{~nm}$. All spectra were recorded in toluene, and toluene was used as a background for all measurements. The photoluminescence emission (PL) spectra were collected using a Cary Eclipse fluorescence spectrophotometer from Varian Instruments with $1 \mathrm{~cm}$ quartz cuvettes. For time-dependent spectroscopy characterization, $10 \boldsymbol{\mu L}$ of crude reaction mixture was diluted with $3 \mathrm{~mL}$ of toluene.

XRD, XPS, FTIR, MALDI-TOF-MS, NMR, and transmission electron microscopy (TEM) Characterizations. Wide-angle powder X-ray diffraction (XRD) was recorded on a Rigaku MiniFlexTM II ( $\mathrm{Cu} \mathrm{K \alpha}$ ) instrument. X-ray photoelectron spectroscopy (XPS) characterization was conducted using a Kratos Axis Ultra DLD system with a Mg anode at $1253.6 \mathrm{eV}$ and Xray power of $150 \mathrm{~W}$. A charge neutralizer was used during the data collection. The survey scans were collected over binding energies of $0-1000 \mathrm{eV}$ with an $80 \mathrm{eV}$ pass energy. For high resolution scans of $\mathrm{Cd}(3 \mathrm{~d})$, $\mathrm{Se}(3 \mathrm{~d})$, and $\mathrm{N}(1 \mathrm{~s})$, a $20 \mathrm{eV}$ pass energy was used. All data were collected so that the $\mathrm{C} 1 \mathrm{~s}$ line was shifted to $284.6 \mathrm{eV}$. At various time points, aliquots of the colloidal synthesis of ligand-passivated (CdSe) $33 / 34$ CSNCs were removed and purified according to the procedure described above. The integrated peak areas of the XPS data yielded the ratio of $\mathrm{Cd}$ to $\mathrm{N}$ at the different time points of the reaction. Based on density functional theory structural prediction, ${ }^{34}$ each magic-sized CdSe CSNC contains $34 \mathrm{Cd}$ atoms. Utilizing this Cd per CSNC number and the integrated XPS areas, we calculated the number of $\mathrm{N}$ atoms per CdSe CSNC and subsequently the number of OLA ligands bound to the surface. The average value was determined from three independent measurements. The result is listed in Table S1. FTIR spectra were acquired using a Thermo Nicolet IS10 FTIR 
spectrometer. For FTIR analysis, samples were prepared using a 1:10 ratio of sample to $\mathrm{KBr}$, ground using a mortar and pestle, and pressed into a pellet. A minimum of 300 scans were collected and all data was processed using Omnic FTIR software. MALDI-TOF-MS analysis was conducted by preparing samples in chloroform by mixing (CdSe) ${ }_{33 / 34} \mathrm{CSNCs}$ and DCTB matrix (1:1000 ratio), drop-casted $5 \mathrm{~mL}$ mixture onto the sample holder and then air-dried at room temperature. The dried samples were analyzed using a Bruker Autoflex MALDI-TOF mass spectrometer equipped with a $\mathrm{N}_{2}$ laser. ${ }^{1} \mathrm{H}$ NMR spectra were recorded on a Bruker Avance III 500 instrument at $500 \mathrm{MHz}$ frequency. Approximately $10 \mathrm{mg}$ of purified OLApassivated (CdSe) ${ }_{33 / 34} \mathrm{CSNCs}$ were dissolved in $\mathrm{CDCl}_{3}$ and a minimum of 1024 scans were collected with $15 \mathrm{~s}$ relaxation delay time and a $30^{\circ}$ pulse angle. At various time points of the synthesis, aliquots were removed and purified according to the procedure described above. 2 $\mu \mathrm{mol}$ of ferrocene was added to each NMR samples as an internal standard in order to determine the concentration of OLA as described in the literature. ${ }^{47,72}$ The average value was determined from three independent measurements. To determine the precise concentration of (CdSe) ${ }_{33 / 34}$ CSNCs in each sample, they were first characterized by UV-vis absorbance spectroscopy and then the absorbance value was used for the concentration calculation by an emperical formula. ${ }^{73}$ The result is listed in Table S2. A similar method was used to

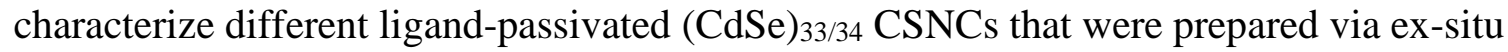
surface exchange procedure. The samples for TEM analysis were prepared by placing $10 \mu \mathrm{L}$ of the CSNCs dissolved in toluene onto a formver-coated copper grid (Electron Microscopy Science). The sample was allowed to set for 30 seconds and any excess solution was removed by wicking with a Kimwipe in order to avoid particle aggregation. Images were acquired using a Techni-12 instrument operating at $100 \mathrm{kV}$. At least 300 CSNCs were analyzed using ImageJ software for the diameter averaging process.

\section{ASSOCIATED CONTENT}

Supporting Information Available. Additional time-dependent UV-visible, photoluminescence, MALDI-TOF-MS, XRD, and XPS spectra, NMR spectra of ligandpassivated CSNCs, TEM images and histograms for size analysis, tables, and molecular diagram. This material is available free of charge via the Internet at http://pubs.acs.org.

\section{AUTHORS INFORMATION}

\section{Corresponding author:}

Rajesh Sardar: rsardar@iupui.edu

Mangilal Agarwal: agarwal@iupui.edu

\section{Author Contributions}

The manuscript was written through contributions of all authors. 


\section{Acknowledgements}

R.S. and M.A acknowledge financial support by IUPUI-OVCR and IUPUI start-up fund, respectively for experimental work. EDX and XRD analyses were conducted with Hitachi and Bruker instruments purchased using NSF-MRI awards (No. MRI-1229514) and (No. MRI1429241), respectively.

\section{REFERENCES}

1. El-Sayed, M. A. Small Is Different: Shape-, Size-, and Composition-Dependent Properties of Some Colloidal Semiconductor Nanocrystals. Acc. Chem. Res. 2004, 37, 326-333.

2. Smith, A. M.; Nie, S. Semiconductor Nanocrystals: Structure, Properties, and Band Gap Engineering. Acc. Chem. Res. 2009, 43, 190-200.

3. Hines, D. A.; Kamat, P. V. Recent Advances in Quantum Dot Surface Chemistry. ACS Appl. Mater. Inter. 2014, 6, 3041-3057.

4. $\quad$ Chen, Z.; Nadal, B.; Mahler, B.; Aubin, H.; Dubertret, B. Quasi-2D Colloidal Semiconductor Nanoplatelets for Narrow Electroluminescence. Adv. Func. Mater. 2014, 24, 295-302.

5. $\quad$ Riehle, F. S.; Bienert, R.; Thomann, R.; Urban, G. A.; Kruger, M. Blue Luminescence and Superstructures from Magic Size Clusters of CdSe. Nano Lett. 2009, 9, 514-518.

6. Alivisatos, A. P. Semiconductor Clusters, Nanocrystals, and Quantum Dots. Science 1996, 271, 933-937.

7. Son, D. H.; Hughes, S. M.; Yin, Y.; Paul Alivisatos, A. Cation Exchange Reactions in Ionic Nanocrystals. Science 2004, 306, 1009-1012.

8. Wang, C.; Shim, M.; Guyot-Sionnest, P. Electrochromic Nanocrystal Quantum Dots. Science 2001, 291, 2390-2392.

9. Dolai, S.; Dutta, P.; Muhoberac, B. B.; Irving, C. D.; Sardar, R. Mechanistic Study of the Formation of Bright White Light-Emitting Ultrasmall CdSe Nanocrystals: Role of Phosphine Free Selenium Precursors. Chem. Mater. 2015, 27, 1057-1070.

10. Kongkanand, A.; Tvrdy, K.; Takechi, K.; Kuno, M.; Kamat, P. V. Quantum Dot Solar Cells. Tuning Photoresponse through Size and Shape Control of CdSe-TiO2 Architecture. J. Am. Chem. Soc. 2008, 130, 4007-4015.

11. Choi, J.-H.; Fafarman, A. T.; Oh, S. J.; Ko, D.-K.; Kim, D. K.; Diroll, B. T.; Muramoto, S.; Gillen, J. G.; Murray, C. B.; Kagan, C. R. Bandlike Transport in Strongly Coupled and Doped Quantum Dot Solids: A Route to High-Performance Thin-Film Electronics. Nano Lett. 2012, 12, 2631-2638.

12. Lee, J.-S.; Kovalenko, M. V.; Huang, J.; Chung, D. S.; Talapin, D. V. Band-like transport, high electron mobility and high photoconductivity in all-inorganic nanocrystal arrays. Nat. Nanotech. 2011, 6, 348-352.

13. Gur, I.; Fromer, N. A.; Geier, M. L.; Alivisatos, A. P. Air-Stable All-Inorganic Nanocrystal Solar Cells Processed from Solution. Science 2005, 310, 462-465.

14. Huynh, W. U.; Dittmer, J. J.; Alivisatos, A. P. Hybrid Nanorod-Polymer Solar Cells. Science 2002, 295, 2425-2427.

15. Kovalenko, M. V.; Scheele, M.; Talapin, D. V. Colloidal Nanocrystals with Molecular Metal Chalcogenide Surface Ligands. Science 2009, 324, 1417-1420.

16. Talapin, D. V.; Murray, C. B. PbSe Nanocrystal Solids for n- and p-Channel Thin Film Field-Effect Transistors. Science 2005, 310, 86-89. 
17. Tisdale, W. A.; Williams, K. J.; Timp, B. A.; Norris, D. J.; Aydil, E. S.; Zhu, X. Y. Hot-Electron Transfer from Semiconductor Nanocrystals. Science 2010, 328, 15431547.

18. Yu, D.; Wang, C.; Guyot-Sionnest, P. n-Type conducting CdSe nanocrystal solids. Science 2003, 300, 1277-1280.

19. LaMer, V. K.; Dinegar, R. H. Theory, Production and Mechanism of Formation of Monodispersed Hydrosols. J. Am. Chem. Soc. 1950, 72, 4847-4854.

20. Krause, M. M.; Mooney, J.; Kambhampati, P. Chemical and Thermodynamic Control of the Surface of Semiconductor Nanocrystals for Designer White Light Emitters. ACS Nano 2013, 7, 5922-5929.

21. Rosson, T. E.; Claiborne, S. M.; McBride, J. R.; Stratton, B. S.; Rosenthal, S. J. Bright White Light Emission from Ultrasmall Cadmium Selenide Nanocrystals. J. Am. Chem. Soc. 2012, 134, 8006-8009.

22. Jin, S.; Harris, R. D.; Lau, B.; Aruda, K. O.; Amin, V. A.; Weiss, E. A. Enhanced rate of radiative decay in cdse quantum dots upon adsorption of an exciton-delocalizing ligand. Nano Lett. 2014, 14, 5323-5328.

23. Bullen, C. R.; Mulvaney, P. Nucleation and growth kinetics of CdSe nanocrystals in octadecene. Nano Lett. 2004, 4, 2303-2307.

24. Qu, L.; Yu, W. W.; Peng, X. In Situ Observation of the Nucleation and Growth of CdSe Nanocrystals. Nano Lett. 2004, 4, 465-469.

25. Hendricks, M. P.; Campos, M. P.; Cleveland, G. T.; Jen-La Plante, I.; Owen, J. S. A tunable library of substituted thiourea precursors to metal sulfide nanocrystals. Science 2015, 348, 1226-1230.

26. Kudera, S.; Zanella, M.; Giannini, C.; Rizzo, A.; Li, Y.; Gigli, G.; Cingolani, R.; Ciccarella, G.; Spahl, W.; Parak, W. J., et al. Sequential Growth of Magic-Size CdSe Nanocrystals. Adv. Mater. 2007, 19, 548-552.

27. Yu, K. CdSe Magic-Sized Nuclei, Magic-Sized Nanoclusters and Regular Nanocrystals: Monomer Effects on Nucleation and Growth. Adv. Mater. 2012, 24, 1123-1132.

28. Cossairt, B. M.; Owen, J. S. CdSe Clusters: At the Interface of Small Molecules and Quantum Dots. Chem. Mater. 2011, 23, 3114-3119.

29. Newton, J. C.; Ramasamy, K.; Mandal, M.; Joshi, G. K.; Kumbhar, A.; Sardar, R. Low-Temperature Synthesis of Magic-Sized CdSe Nanoclusters: Influence of Ligands on Nanocluster Growth and Photophysical Properties. J. Phys. Chem. C 2012, 116, 4380-4389.

30. Kilina, S.; Velizhanin, K. A.; Ivanov, S.; Prezhdo, O. V.; Tretiak, S. Surface Ligands Increase Photoexcitation Relaxation Rates in CdSe Quantum Dots. ACS Nano 2012, 6, 6515-6524.

31. Kilina, S.; Ivanov, S.; Tretiak, S. Effect of Surface Ligands on Optical and Electronic Spectra of Semiconductor Nanoclusters. J. Am. Chem. Soc. 2009, 131, 7717-7726.

32. Dolai, S.; Nimmala, P. R.; Mandal, M.; Muhoberac, B. B.; Dria, K.; Dass, A.; Sardar, R. Isolation of Bright Blue Light-Emitting CdSe Nanocrystals with $6.5 \mathrm{kDa}$ Core in Gram Scale: High Photoluminescence Efficiency Controlled by Surface Ligand Chemistry. Chem. Mater. 2014, 26, 1278-1285.

33. Wang, Y.; Zhang, Y.; Wang, F.; Giblin, D. E.; Hoy, J.; Rohrs, H. W.; Loomis, R. A.; Buhro, W. E. The Magic-Size Nanocluster (CdSe)34 as a Low-Temperature Nucleant for Cadmium Selenide Nanocrystals; Room-Temperature Growth of Crystalline Quantum Platelets. Chem. Mater. 2014, 26, 2233-2243.

34. Kasuya, A.; Sivamohan, R.; Barnakov, Y. A.; Dmitruk, I. M.; Nirasawa, T.; Romanyuk, V. R.; Kumar, V.; Mamykin, S. V.; Tohji, K.; Jeyadevan, B., et al. Ultra- 
stable nanoparticles of CdSe revealed from mass spectrometry. Nat. Mater. 2004, 3, 99-102.

35. Harrell, S. M.; McBride, J. R.; Rosenthal, S. J. Synthesis of Ultrasmall and MagicSized CdSe Nanocrystals. Chem. Mater. 2013, 25, 1199-1210.

36. Sowers, K. L.; Swartz, B.; Krauss, T. D. Chemical Mechanisms of Semiconductor Nanocrystal Synthesis. Chem. Mater. 2013, 25, 1351-1362.

37. Schaaff, T. G.; Knight, G.; Shafigullin, M. N.; Borkman, R. F.; Whetten, R. L. Isolation and Selected Properties of a $10.4 \mathrm{kDa}$ Gold:Glutathione Cluster Compound. J. Phys. Chem. B 1998, 102, 10643-10646.

38. Jin, R.; Zeng, C.; Zhou, M.; Chen, Y. Atomically Precise Colloidal Metal Nanoclusters and Nanoparticles: Fundamentals and Opportunities. Chem. Rev. 2016, 116, 10346-10413.

39. Dass, A. Mass Spectrometric Identification of Au68(SR)34 Molecular Gold Nanoclusters with 34-Electron Shell Closing. J. Am. Chem. Soc. 2009, 131, 1166611667.

40. Qian, H.; Zhu, Y.; Jin, R. Isolation of Ubiquitous Au40(SR)24 Clusters from the 8 kDa Gold Clusters. J. Am. Chem. Soc. 2010, 132, 4583-4585.

41. Xie, L.; Shen, Y.; Franke, D.; Sebastián, V.; Bawendi, M. G.; Jensen, K. F. Characterization of Indium Phosphide Quantum Dot Growth Intermediates Using MALDI-TOF Mass Spectrometry. J. Am. Chem. Soc. 2016, 138, 13469-13472.

42. Kim, B. H.; Shin, K.; Kwon, S. G.; Jang, Y.; Lee, H.-S.; Lee, H.; Jun, S. W.; Lee, J.; Han, S. Y.; Kim, D.-H., et al. Sizing by weighing: characterizing sizes of ultrasmallsized iron oxide nanocrystals using MALDI-TOF mass spectrometry. J. Am. Chem. Soc. 2013, 135, 2407-2410.

43. Wang, Y.; Liu, Y.-H.; Zhang, Y.; Wang, F.; Kowalski, P. J.; Rohrs, H. W.; Loomis, R. A.; Gross, M. L.; Buhro, W. E. Isolation of the Magic-Size CdSe Nanoclusters [(CdSe)13(n-octylamine)13] and [(CdSe)13(oleylamine)13]. Angew. Chem. Int. Ed. 2012, 51, 6154-6157.

44. Chen, X.; Samia, A. C. S.; Lou, Y.; Burda, C. Investigation of the Crystallization Process in 2 nm CdSe Quantum Dots. J. Am. Chem. Soc. 2005, 127, 4372-4375.

45. Katari, J. B.; Colvin, V. L.; Alivisatos, A. P. X-ray photoelectron spectroscopy of CdSe nanocrystals with applications to studies of the nanocrystal surface. J. Phys. Chem. 1994, 98, 4109-4117.

46. Teunis, M. B.; Dolai, S.; Sardar, R. Effects of Surface-Passivating Ligands and Ultrasmall CdSe Nanocrystal Size on the Delocalization of Exciton Confinement. Langmuir 2014, 30, 7851-7858.

47. Anderson, N. C.; Hendricks, M. P.; Choi, J. J.; Owen, J. S. Ligand Exchange and the Stoichiometry of Metal Chalcogenide Nanocrystals: Spectroscopic Observation of Facile Metal-Carboxylate Displacement and Binding. J. Am. Chem. Soc. 2013, 135, 18536-18548.

48. Moreels, I.; Fritzinger, B.; Martins, J. C.; Hens, Z. Surface Chemistry of Colloidal PbSe Nanocrystals. J. Am. Chem. Soc. 2008, 130, 15081-15086.

49. Lawrence, K. N.; Dutta, P.; Nagaraju, M.; Teunis, M. B.; Muhoberac, B. B.; Sardar, R. Dual Role of Electron-Accepting Metal-Carboxylate Ligands: Reversible Expansion of Exciton Delocalization and Passivation of Nonradiative Trap-States in Moleculelike CdSe Nanocrystals. J. Am. Chem. Soc. 2016, 138, 12813-12825.

50. Morris-Cohen, A. J.; Vasilenko, V.; Amin, V. A.; Reuter, M. G.; Weiss, E. A. Model for adsorption of ligands to colloidal quantum dots with concentration-dependent surface structure. ACS Nano 2011, 6, 557-565.

51. Bullen, C.; Mulvaney, P. The effects of chemisorption on the luminescence of CdSe quantum dots. Langmuir 2006, 22, 3007-3013. 
52. Ji, X.; Copenhaver, D.; Sichmeller, C.; Peng, X. Ligand bonding and dynamics on colloidal nanocrystals at room temperature: the case of alkylamines on CdSe nanocrystals. J. Am. Chem. Soc. 2008, 130, 5726-5735.

53. Murray, C. B.; Norris, D. J.; Bawendi, M. G. Synthesis and characterization of nearly monodisperse CdE ( $\mathrm{E}=$ sulfur, selenium, tellurium) semiconductor nanocrystallites. $J$. Am. Chem. Soc. 1993, 115, 8706-8715.

54. Pradhan, N.; Reifsnyder, D.; Xie, R.; Aldana, J.; Peng, X. Surface Ligand Dynamics in Growth of Nanocrystals. J. Am. Chem. Soc. 2007, 129, 9500-9509.

55. Dannhauser, T.; O'Neil, M.; Johansson, K.; Whitten, D.; McLendon, G. Photophysics of quantized colloidal semiconductors. Dramatic luminescence enhancement by binding of simple amines. J. Phys. Chem. 1986, 90, 6074-6076.

56. Knowles, K. E.; Tice, D. B.; McArthur, E. A.; Solomon, G. C.; Weiss, E. A. Chemical control of the photoluminescence of CdSe quantum dot- organic complexes with a series of para-substituted aniline ligands. J. Am. Chem. Soc. 2009, 132, 1041-1050.

57. Medintz, I. L.; Uyeda, H. T.; Goldman, E. R.; Mattoussi, H. Quantum dot bioconjugates for imaging, labelling and sensing. Nat. Mater. 2005, 4, 435-446.

58. Bruchez, M.; Moronne, M.; Gin, P.; Weiss, S.; Alivisatos, A. P. Semiconductor Nanocrystals as Fluorescent Biological Labels. Science 1998, 281, 2013-2016.

59. Chan, W. C. W.; Nie, S. Quantum Dot Bioconjugates for Ultrasensitive Nonisotopic Detection. Science 1998, 281, 2016-2018.

60. Kolb, H. C.; Finn, M. G.; Sharpless, K. B. Click Chemistry: Diverse Chemical Function from a Few Good Reactions. Angew. Chem. Int. Ed. 2001, 40, 2004-2021.

61. Connon, S. J.; Blechert, S. Recent Developments in Olefin Cross-Metathesis. Angew. Chem. Int. Ed. 2003, 42, 1900-1923.

62. Tavasoli, E.; Guo, Y.; Kunal, P.; Grajeda, J.; Gerber, A.; Vela, J. Surface Doping Quantum Dots with Chemically Active Native Ligands: Controlling Valence without Ligand Exchange. Chem. Mater. 2012, 24, 4231-4241.

63. Selvan, S. T.; Tan, T. T.; Ying, J. Y. Robust, Non-Cytotoxic, Silica-Coated CdSe Quantum Dots with Efficient Photoluminescence. Adv. Mater. 2005, 17, 1620-1625.

64. Liu, H.; Espe, M.; Modarelli, D. A.; Arias, E.; Moggio, I.; Ziolo, R. F.; Heinz, H. Interaction of substituted poly(phenyleneethynylene)s with ligand-stabilized CdS nanoparticles. J. Mater. Chem. A 2014, 2, 8705-8711.

65. Weiss, P. S. Functional Molecules and Assemblies in Controlled Environments: Formation and Measurements. Acc. Chem. Res. 2008, 41, 1772-1781.

66. Joshi, G. K.; Blodgett, K. N.; Muhoberac, B. B.; Johnson, M. A.; Smith, K. A.; Sardar, R. Ultrasensitive Photoreversible Molecular Sensors of Azobenzene-Functionalized Plasmonic Nanoantennas. Nano Lett. 2014, 14, 532-540.

67. Frederick, M. T.; Amin, V. A.; Swenson, N. K.; Ho, A. Y.; Weiss, E. A. Control of Exciton Confinement in Quantum Dot-Organic Complexes through Energetic Alignment of Interfacial Orbitals. Nano Lett. 2012, 13, 287-292.

68. Lieber, E.; Rao, C. N. R.; Chao, T. S.; Hoffman, C. W. W. Infrared Spectra of Organic Azides. Anal. Chem. 1957, 29, 916-918.

69. Tecklenburg, M. M. J.; Kosnak, D. J.; Bhatnagar, A.; Mohanty, D. K. Vibrational characterization of azobenzenes, azoxybenzenes and azoaromatic and azoxyaromatic polyethers. J. Raman Spectro. 1997, 28, 755-763.

70. Nagao, Y.; Takasu, A. "Click polyester”: Synthesis of polyesters containing triazole units in the main chain via safe and rapid "click" chemistry and their properties. $J$. Poly. Sci. Part A: Poly. Chem. 2010, 48, 4207-4218.

71. Lin, H.-Y.; Snider, B. B. Synthesis of Phidianidines A and B. J. Org. Chem. 2012, 77, 4832-4836. 
72. Dolai, S.; Dass, A.; Sardar, R. Photophysical and Redox Properties of Molecule-like CdSe Nanoclusters. Langmuir 2013, 29, 6187-6193.

73. Yu, W. W.; Qu, L.; Guo, W.; Peng, X. Experimental Determination of the Extinction Coefficient of CdTe, CdSe, and CdS Nanocrystals. Chem. Mater. 2003, 15, 2854-2860. 


\section{LIST OF FIGURES}

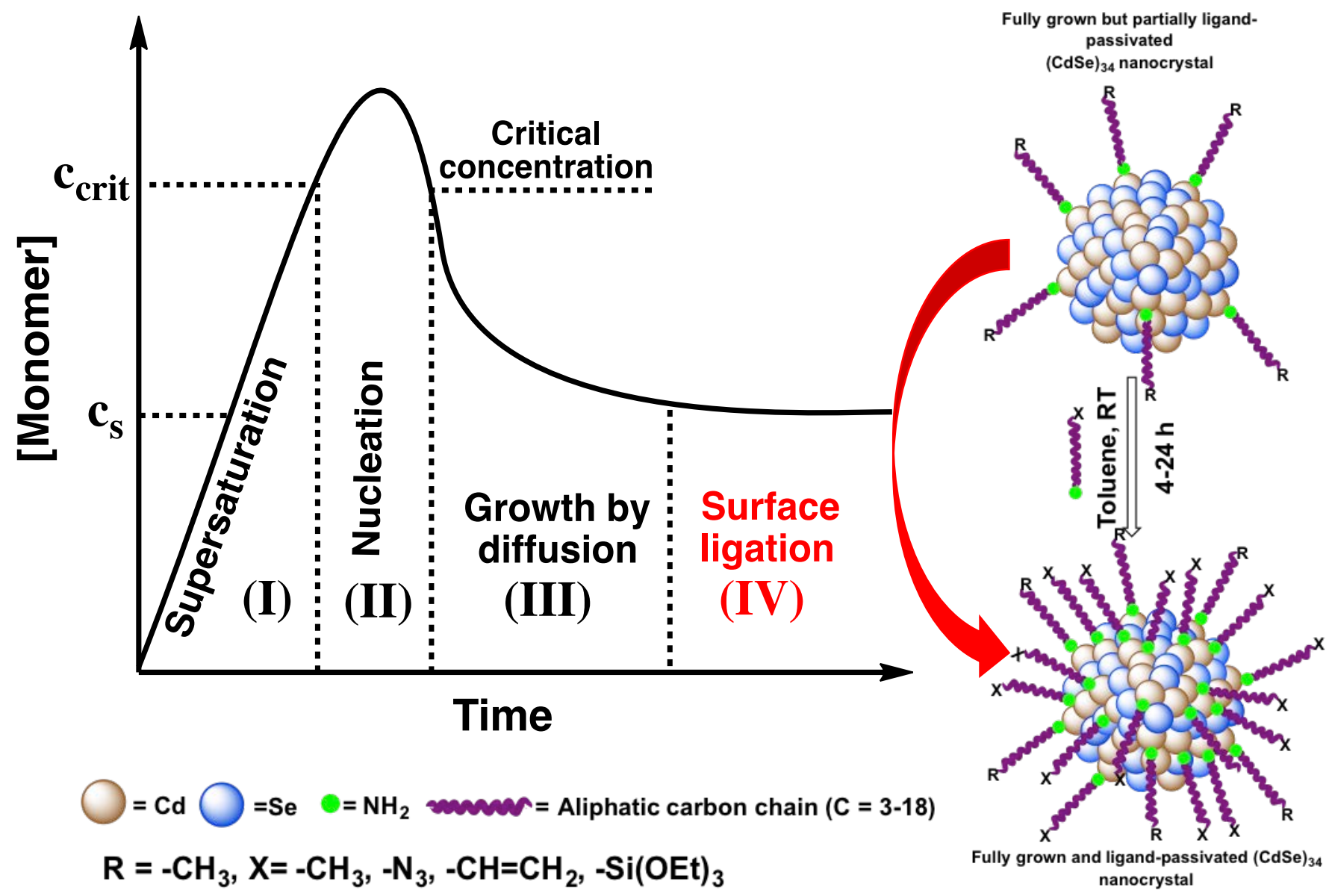

Scheme 1. (Left) General mechanism of colloidal CdSe CSNC formation showing a clearly distinguishable step (IV) of surface ligand attachment onto fully-grown nancrystals. (Right) Schematic representation of the additional ligand attachment onto partially ligand-passivated (CdSe) $)_{33 / 34}$ CSNCs as part of step (IV) is shown in red curved arrow. 

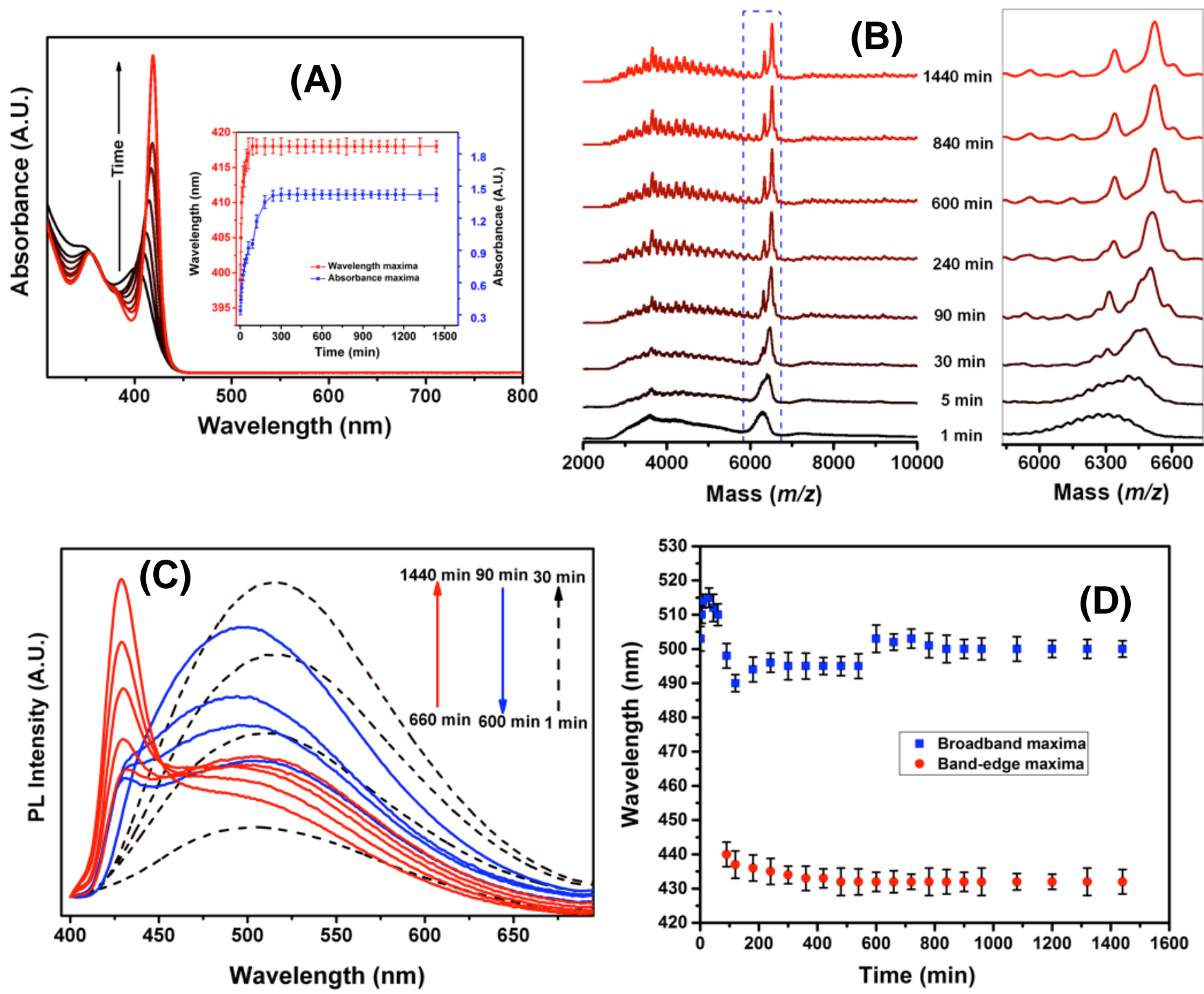

Figure 1. (A) Representative time-dependent UV-vis absorption spectra of (CdSe) ${ }_{33 / 34}$ CSNC formation at room temperature. The inset shows position and intensity of lowest energy absorption peak over time. (B) MALDI-TOF-MS spectra acquired at different time point during the nucleation and growth of (CdSe) $33 / 34$ CSNCs. Expanded spectra are shown in black edge box. (C) Time-dependent PL spectra of CdSe CSNCs at room temperature. (D) Changes in the broadband (blue squares) and band-edge (red dots) PL position maxima during the formation of (CdSe) $)_{33 / 34}$ CSNCs at room temperature over the course of $24 \mathrm{~h}$. For UV-vis and PL measurements, crude reaction mixture was diluted with toluene before acquiring the spectra. 

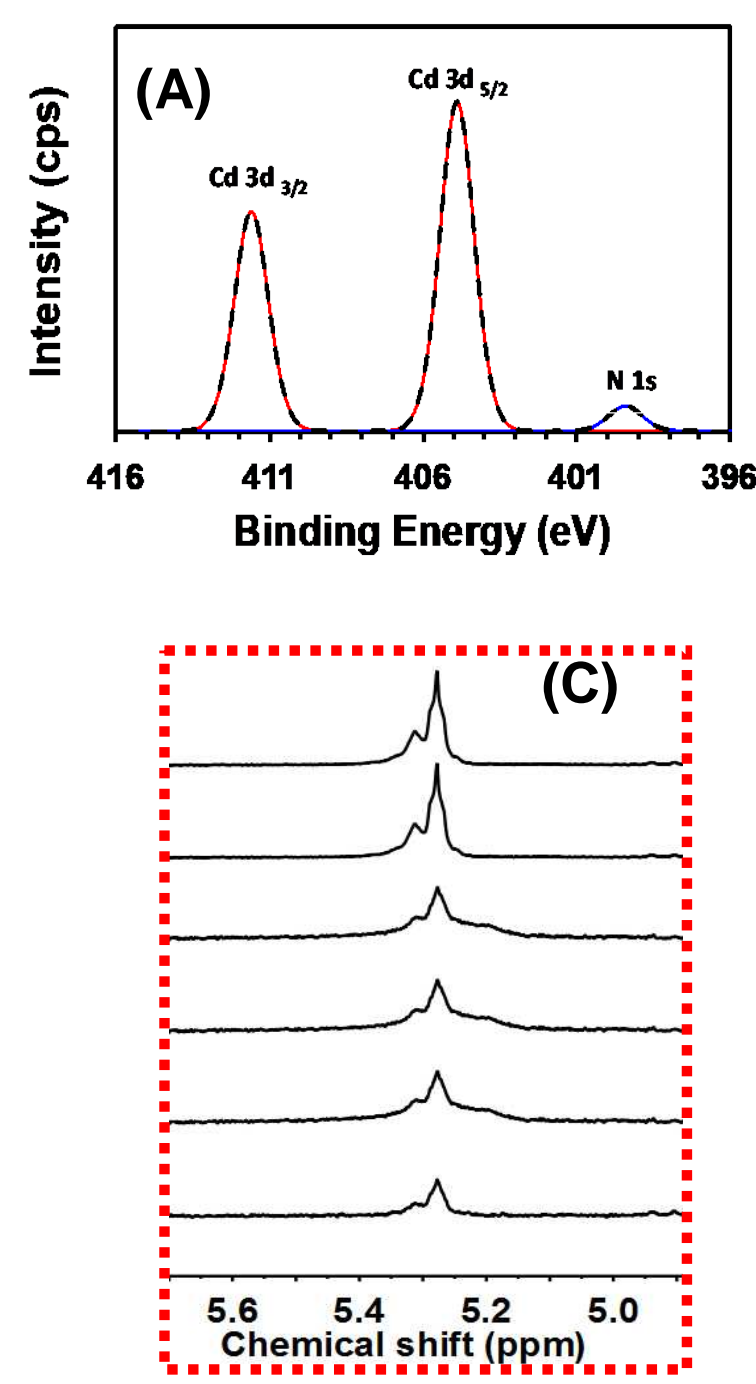
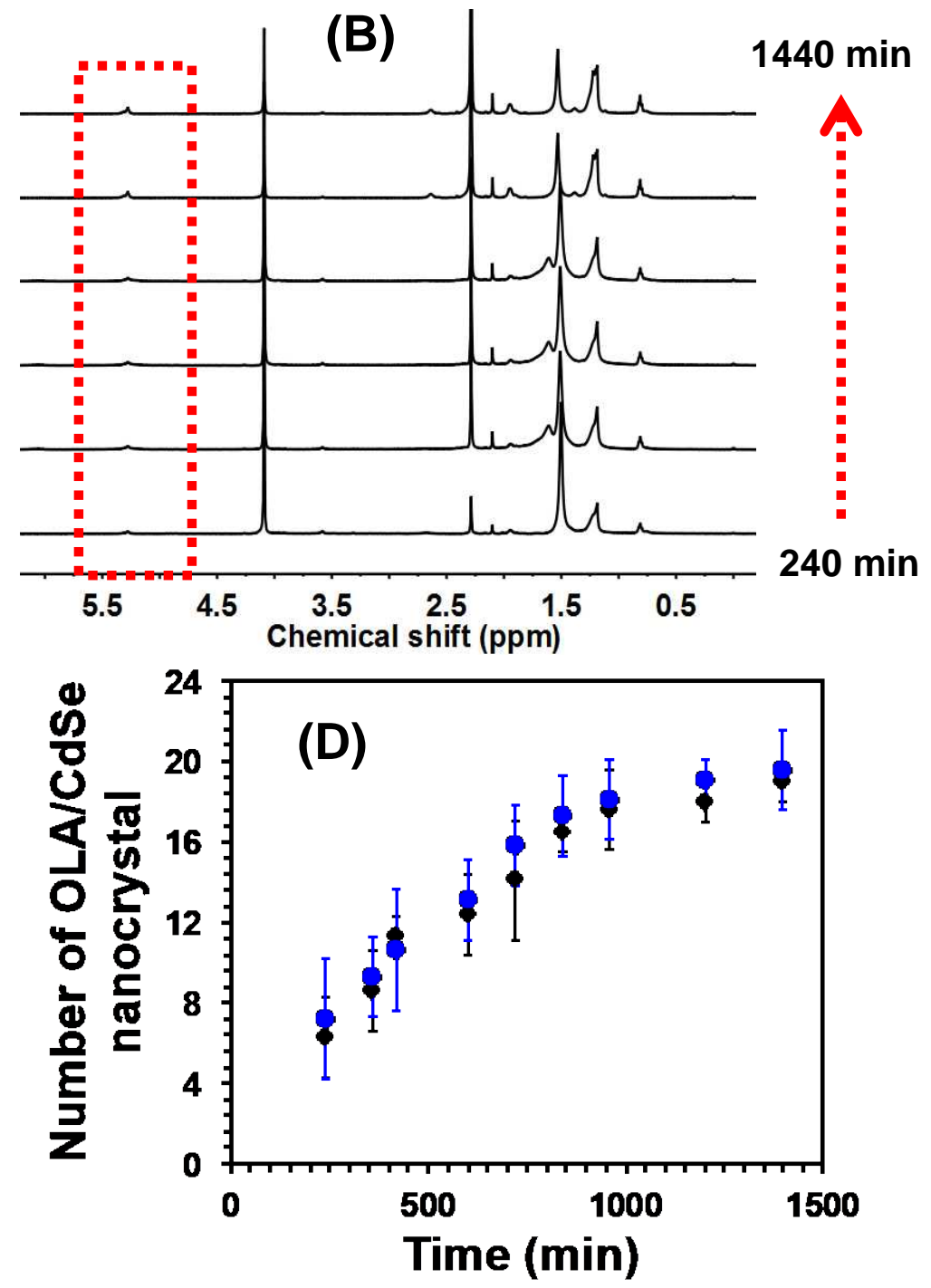

Figure 2. (A) XPS spectrum of fully OLA-passivated (CdSe) $33 / 34$ CSNCs. The dotted line shows fits to the Cd 3D and N 1s peaks. (B) Time-dependent ${ }^{1} \mathrm{H}$ NMR spectra of (CdSe) $)_{34}$ CSNCs at room temperature. All spectra were recorded in $\mathrm{CDCl}_{3}$. The dotted red box represents the vinyl region of OLA as shown in the expanded spectra of panel C. (D) Plot of number of OLAs per (CdSe) ${ }_{33 / 34}$ CSNCs in purified samples over time determined from XPS (black diamonds) and NMR (blue dots) analyses from three independent measurements. We considered the (CdSe) $)_{33 / 34}$ CSNC core fully grown by $240 \mathrm{~min}$. 

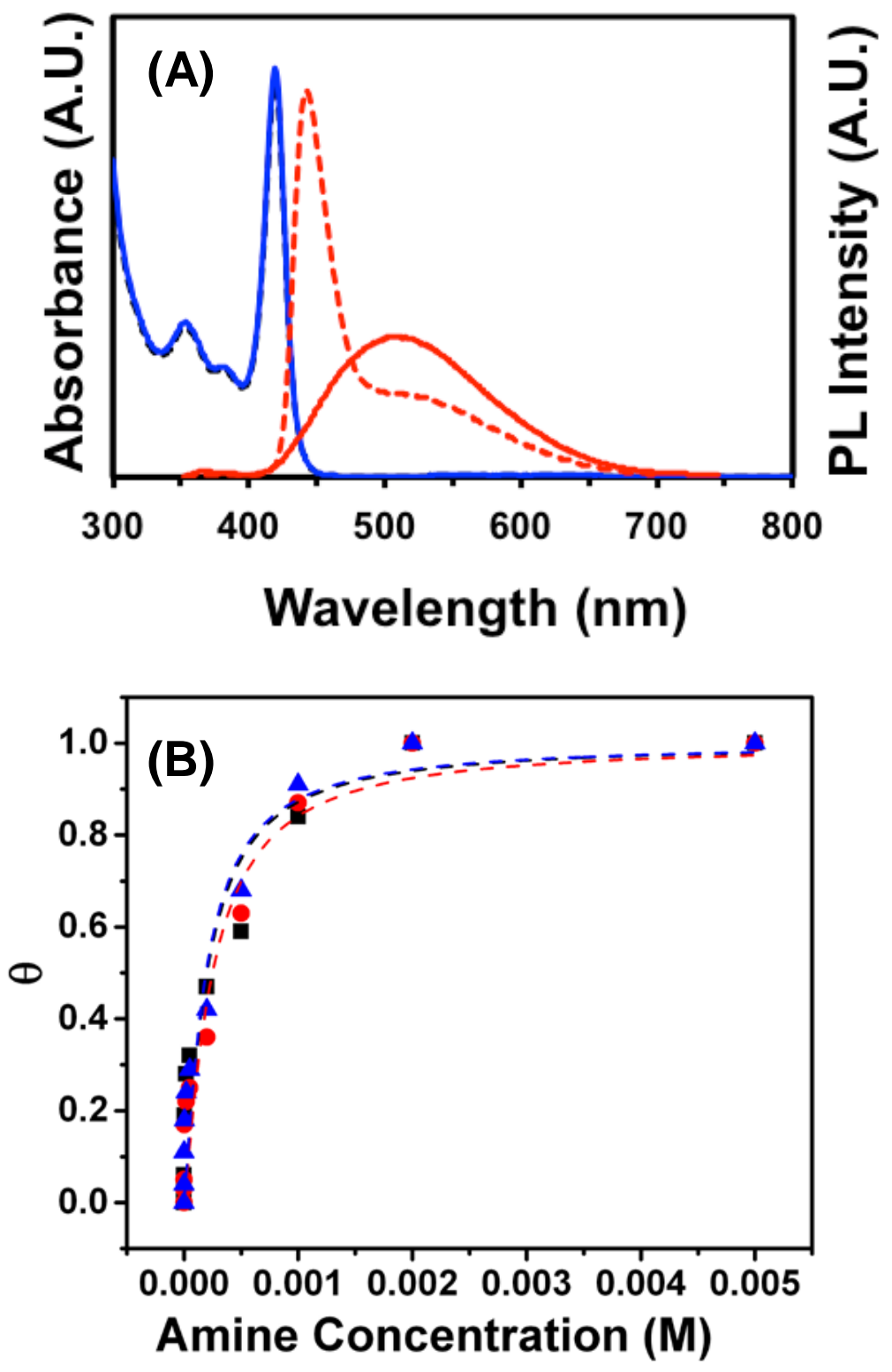

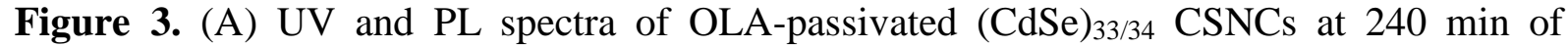
reaction after purification (solid blue and red lines, respectively) and after ex-situ OLA treatment for $24 \mathrm{~h}$ (dotted black and red lines, respectively). (B) Fraction of surface coverage of the ligand (OLA, red dots; DCA, black squares; and OCA, blue triangles) as a function of their amine concentration for $0.12 \mathrm{mM}(\mathrm{CdSe})_{34} \mathrm{CSNCs}$. The dotted lines are separated fits to the Langmuir isotherm using Eq. 1. 


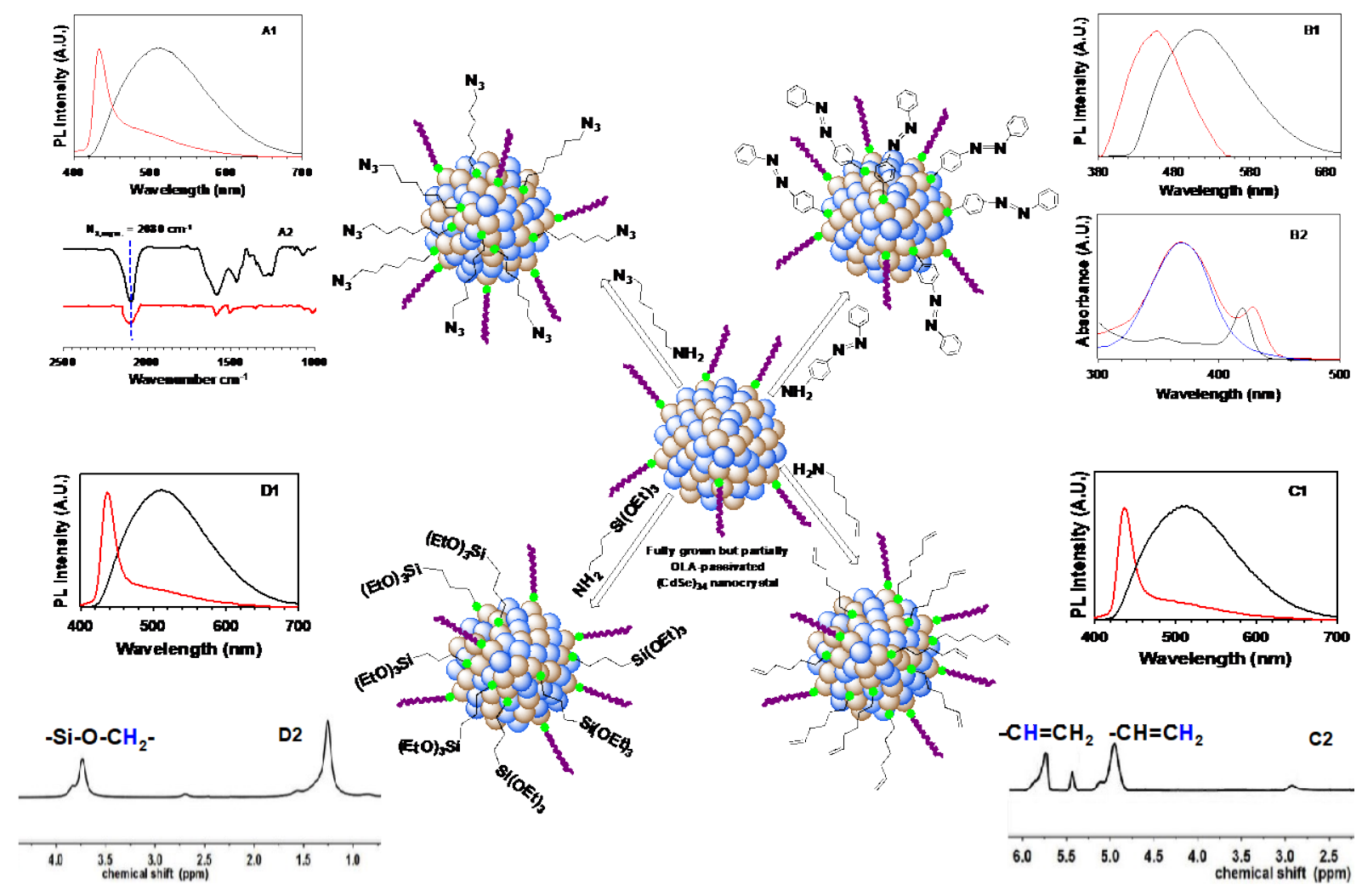

Figure 4. (A) Schematic diagram and experimental results of mixed ligand-passivated (CdSe) ${ }_{33 / 34}$ CSNCs. (A1) PL spectra of (black) OLA- and (red) mixed OLA-/AHA-passivated (CdSe) ${ }_{33 / 34}$ CSNCs. (A2) FTIR spectra of (CdSe) ${ }_{33 / 34}$ CSNCs with the same ligation as A1 and the same color scheme. (B1) PL and (B2) UV-vis spectra of (black) OLA- and (red) mixed OLA-/AAB-passivated (CdSe) ${ }_{33 / 34}$ CSNCs. The blue curve in B2 centered at $~ 365 \mathrm{~nm}$ represents the UV-vis spectrum of azobenzene in the trans confirmation. ${ }^{65}$ (C1) PL of (black) only OLA- and (red) mixed OLA-/HXA-passivated (CdSe) ${ }_{33 / 34}$ CSNCs. (C2) ${ }^{1} \mathrm{H}$ NMR spectrum of mixed OLA-/HXA-passivated (CdSe) ${ }_{33 / 34}$ CSNCs. Peaks at 5.83 and 4.88 ppm represent $-\mathrm{CH}=\mathrm{CH}$ resonances of $\mathrm{HXA}$. The peak at $5.34 \mathrm{ppm}$ originates from $-\mathrm{CH}=\mathrm{CH}-$ resonance of OLA. (D1) PL of (black) only OLA- and (red) mixed OLA-/APTES-passivated (CdSe) ${ }_{33 / 34}$ CSNCs. (D2) ${ }^{1} \mathrm{H}$ NMR spectrum of mixed OLA-/APTES-passivated (CdSe) $33 / 34$ CSNCs. Peak at 3.83 ppm represents -Si-O-CH2- resonances of APTES. Full NMR spectra are provided in the supporting information file. 

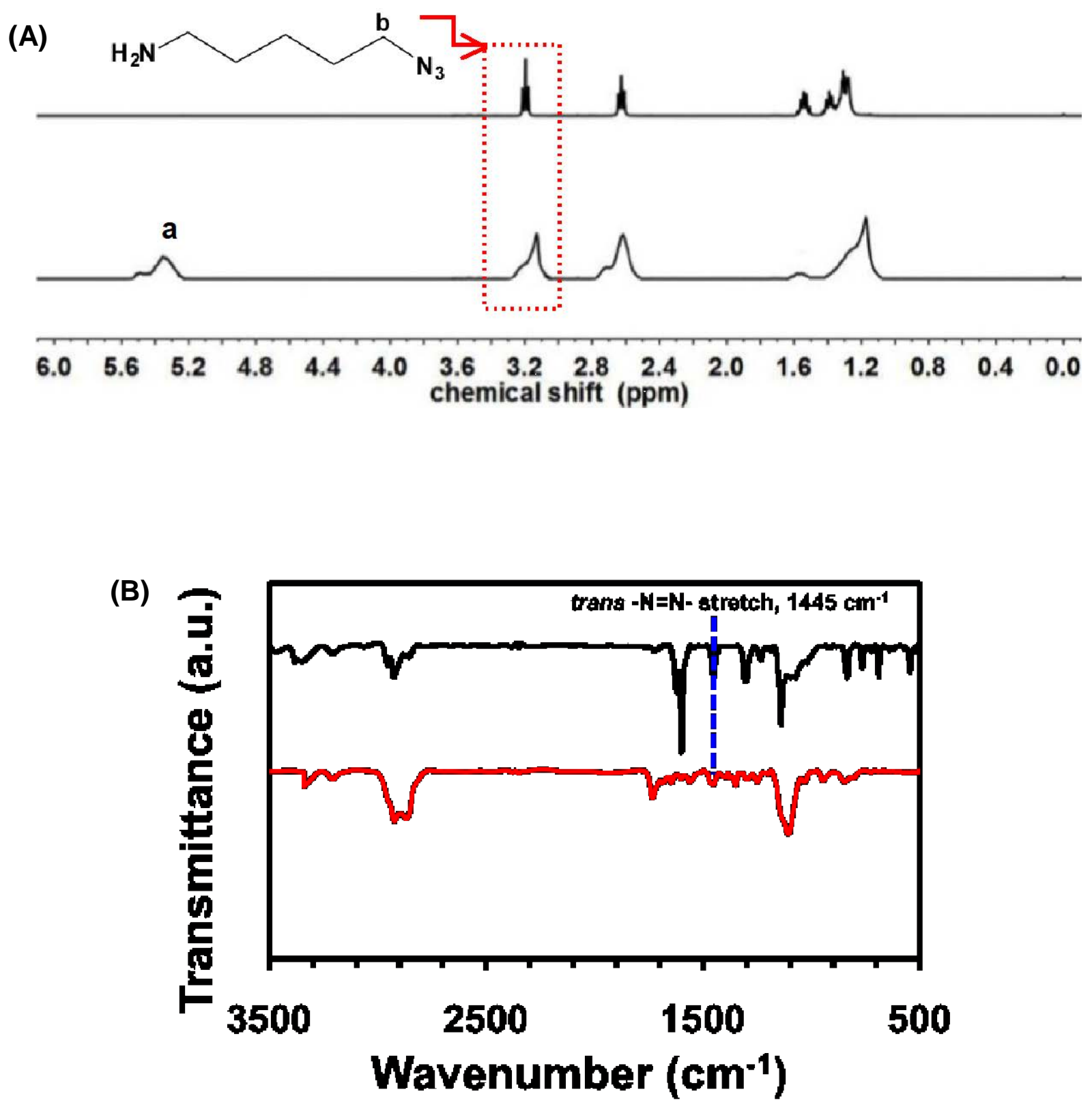

Figure 5. (A) ${ }^{1} \mathrm{H}$ NMR spectrum of mixed OLA-/AHA-passivated (CdSe) ${ }_{33 / 34}$ CSNCs (bottom). The characteristic vinyl (a, $-\mathrm{CH}=\mathrm{CH}-)$ resonance of OLA at $5.34 \mathrm{ppm}$ and methylene resonance of AHA adjuscent to azide (b, $-\mathrm{CH}_{2}-\mathrm{N}_{3}$ ) at $3.21 \mathrm{ppm}$, repectively, observed. The NMR peak broadening is because of spin-spin relaxation and dipole broadening from surface bound ligands. A sharp resonance from $-\mathrm{CH}_{2}-\mathrm{N}_{3}$ is observed for pure AHA (top). The NMR data equivocally prove the mixed surface ligation of (CdSe)33/34 CSNCs. (B) FTIR spectra of pure AAB (black) and mixed OLA-/AAB-passivated (CdSe) $33 / 34$ CSNCs (red). Dotted blue line indicates the $-\mathrm{N}=\mathrm{N}$ - stretch of $\mathrm{AB}$ when it is in the trans conformation. ${ }^{69}$ 


\section{$\underline{\text { TOC Graphic }}$}

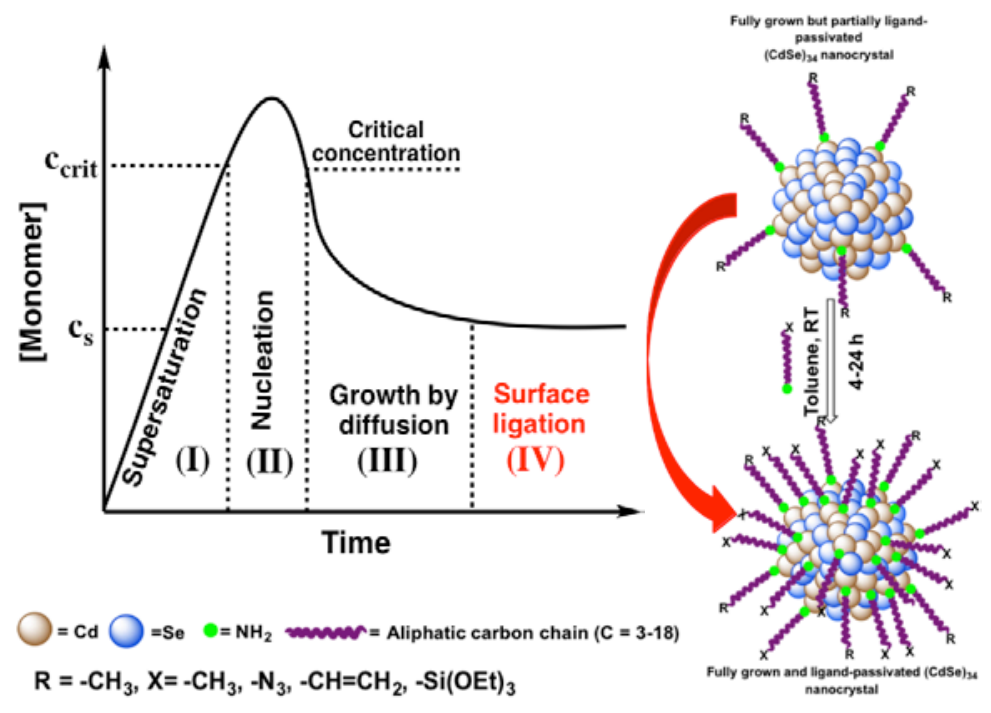

